\title{
Failure analysis of SA210-C steel pipe in power plant
}

\author{
Hai Zhao ${ }^{1, *}$, Chong Jiang ${ }^{1}, Z^{2}$ hiwei Gao ${ }^{1}$ and Chengchuan Tian ${ }^{1}$ \\ ${ }^{1}$ Huadian Electric Power Research Institute Co., LTD, Hangzhou, Zhejiang, 310030, China
}

\begin{abstract}
The reason and mechanism of the failure of SA210-C steel liquid wall were analyzed by means of macro morphology analysis, chemical composition analysis, microstructure analysis and XRD phase analysis. The test results show that the main reason for the failure of SA210-C steel water wall tube is the corrosion under the inner wall scale, and the long-term unqualified boiler water quality is the main factor causing the corrosion of water wall.
\end{abstract}

\section{Introduction}

In recent years, non-stop accidents caused by corrosion under scale frequently occur in thermal power generating units. Due to the poor thermal conductivity of scale, the temperature of the places where the deposits are attached to the metal wall surface rises sharply. When the temperature rises to a certain extent, it will exceed the bearing limit of the metal, that is, the surface temperature of the metal is higher than the allowable temperature, and the metal will creep because of the high temperature, resulting in the reduction of the strength of the metal. Under bad working conditions, the boiler is prone to bulge, perforation, rupture and other phenomena at the location of corrosion under scale, which can easily cause leakage of heating surface, hinder the normal operation of the unit, and even cause serious consequences such as non-stop of the unit ${ }^{[1-3]}$.

As one of the common failure forms of boilers in use, it is of great significance for our production to understand its mechanism ${ }^{[4]}$. In this paper, the type of corrosion and the specific cause of corrosion are studied through the analysis of the sampling test of scaling corrosion water wall tube in a power station.

\section{Test and analysis}

\subsection{Macro morphology analysis}

During the minor repair of the thermal power unit, corrosion leakage of the water wall was found, and a section of pipe sample was cut in the serious corrosion area for the test in this paper. This section of water wall pipe is internally threaded pipe, which was put into production in 2008, material: SA-210C, specification: $\Phi 63.5 \times 7 \mathrm{~mm}$, flue gas temperature: $797^{\circ} \mathrm{C}$, medium temperature: $366^{\circ} \mathrm{C}$, pressure: $20.21 \mathrm{MPa}$.

Fig. 1 is a macro photo of the back fire surface and the front fire surface of the water wall tube. It can be seen that the water wall tube diameter has no obvious expansion, the outer wall of the tube has no cracks, damage and other macro defects, and there is no serious outer wall oxidation.

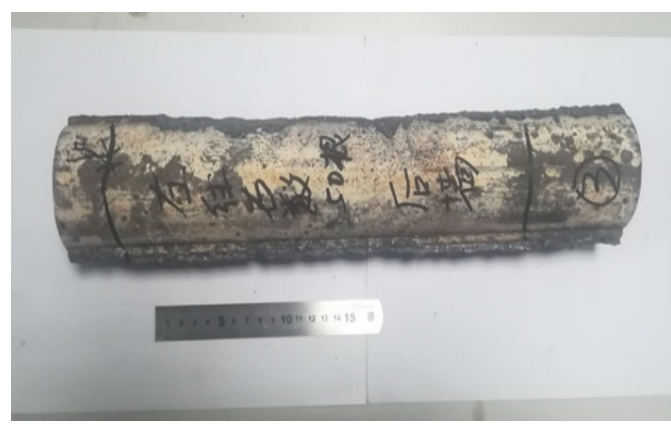

Fig. 1. Macroscopic appearance of water wall tube

When the water wall tube is cut open, it is found that there is corrosion on the inner wall of the fire facing surface of the tube. The corrosion is multiple corrosion pits in a strip, and the black and dense scale layer is covered on the corrosion pits, as shown in Figure 2.

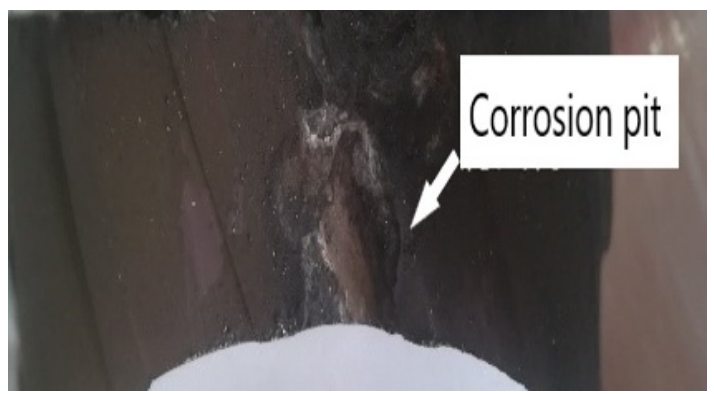

Fig. 2. Corrosion of inner wall of water wall tube

\subsection{Chemical composition analysis}

A $25 \mathrm{~mm} \times 25 \mathrm{~mm} \times 3 \mathrm{~mm}$ metal sheet is cut from the water wall tube, and the composition is determined by spark direct reading spectrometer. The results are shown in Table 1. Compared with ASME sa-210 / sa-210m "medium carbon steel tubes for boiler and Superheater" [5], 
the chemical composition of water wall tubes meets the standard composition.

Table1. Chemical composition analysis results of water wall tube (mass\%)

\begin{tabular}{|c|c|c|c|c|c|c|}
\hline Element & $\mathbf{C}$ & $\mathbf{S i}$ & $\mathbf{M n}$ & $\mathbf{S}$ & $\mathbf{P}$ & $\mathbf{F e}$ \\
\hline Sample & 0.232 & 0.234 & 0.910 & 0.0019 & 0.0028 & Allowance \\
\hline Eligibility criteria & $\leqslant 0.35$ & $\geqslant 0.10$ & $0.29 \sim 1.06$ & $\leqslant 0.035$ & $\leqslant 0.035$ & Allowance \\
\hline
\end{tabular}

\subsection{Mechanical property test}

The tensile specimens are cut along the length direction of the water wall tube toward the fire surface and back fire surface, and the tensile test is carried out with Ag xplus $100 \mathrm{kN}$ universal material testing machine. The tensile test results are shown in Table 2. Compared with ASME sa$210 / \mathrm{sa}-210 \mathrm{~m}$ medium carbon steel tubes for boiler and Superheater, the yield strength of the fire facing and back facing surfaces of the water wall tubes are lower than the standard requirements, and the tensile strength and elongation at break meet the standard requirements, but the tensile strength test results of the fire facing surfaces are only slightly higher than the lower limit of the qualified standard ${ }^{[5]}$.

Table2. Mechanical property test results

\begin{tabular}{|c|c|c|c|}
\hline \multirow{2}{*}{ Sample } & \multicolumn{3}{|c|}{ Mechanical property } \\
\cline { 2 - 4 } & $\begin{array}{c}\text { Rp0.2 } \\
\text { (MPa) }\end{array}$ & $\begin{array}{c}\text { Rm } \\
\text { (MPa) }\end{array}$ & $\begin{array}{c}\text { Elongation } \\
\text { (\%) }\end{array}$ \\
\hline Fireside & 162.8 & 490.8 & 35.86 \\
\hline $\begin{array}{c}\text { Back fire } \\
\text { surface }\end{array}$ & 221.9 & 534.4 & 37.08 \\
\hline $\begin{array}{c}\text { Eligibility } \\
\text { criteria }\end{array}$ & $\geqslant 275$ & $\geqslant 485$ & $\geqslant 30$ \\
\hline
\end{tabular}

\subsection{Microstructure observation}

Figure 3 shows the micro morphology of the corrosion pit of the water wall tube. It is observed from the figure that the corrosion products and scale layers are successively covered above the base metal of the corrosion pit, and some of the corrosion products and scale have fallen off. The so-called scale refers to the solid attachment on the metal surface of the water side of the heating surface in the boiler with the water temperature rising or evaporation concentration after the impurities carried in the feed water enter the boiler ${ }^{[3,6]}$.

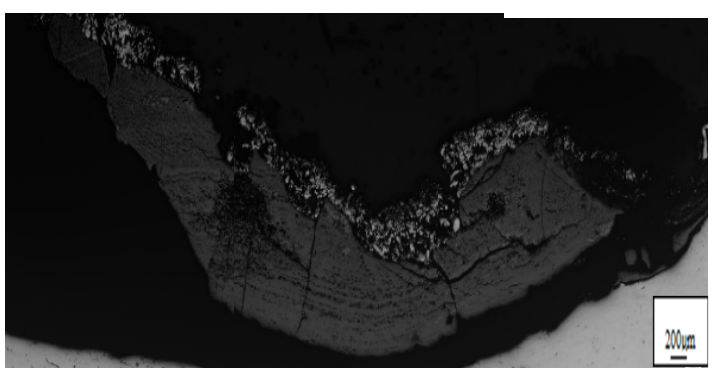

Fig. 3. Scale corrosion layer of sample

The metallographic specimen was prepared at the corrosion site of the pipe. Fig. 4 shows the micro morphology of the pipe section under the condition that the sample is not etched. The residual thickness of the pipe wall after corrosion is $4.88 \mathrm{~mm}$, and the wall thickness is reduced by $30.3 \%$. The No. 1 position in the figure is close to the corrosion pit, and there are dense microcracks within $1.69 \mathrm{~mm}$ from the corrosion pit to the inside of the pipe.

Continue to observe the position 1 in Fig. 4 at a higher multiple, where the microcrack is intergranular, and no defects are found at other positions (Fig. 5).

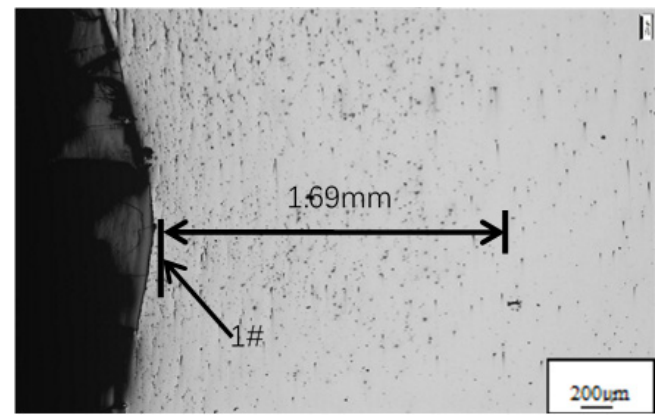

Fig. 4. Micro morphology of sample

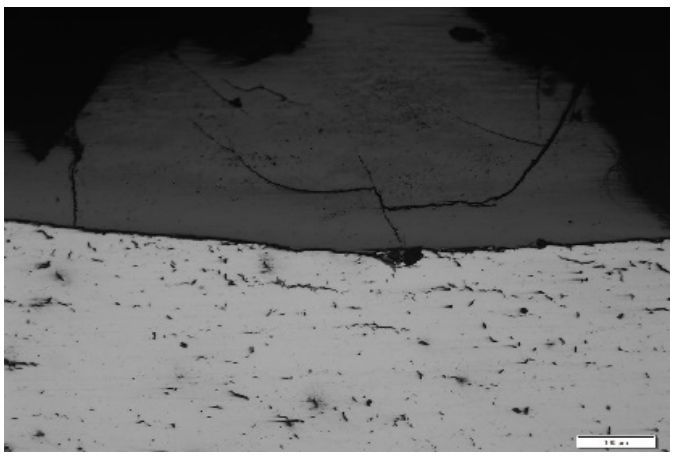

Fig. 5. 1\#Micro morphology of sample 
Fig. 6 shows the metallographic structure of each part of the sample after etching. The metallographic structure of the corrosion pit (inner wall) and the part far away from the corrosion pit are ferrite and pearlite, and there is no sign of serious spheroidization and other structural abnormalities ${ }^{[7]}$, but there are microcracks at the edge of the corrosion pit (Fig. 6a). Compared with the other two parts, the pearlite area is reduced, and there is obvious decarburization phenomenon.

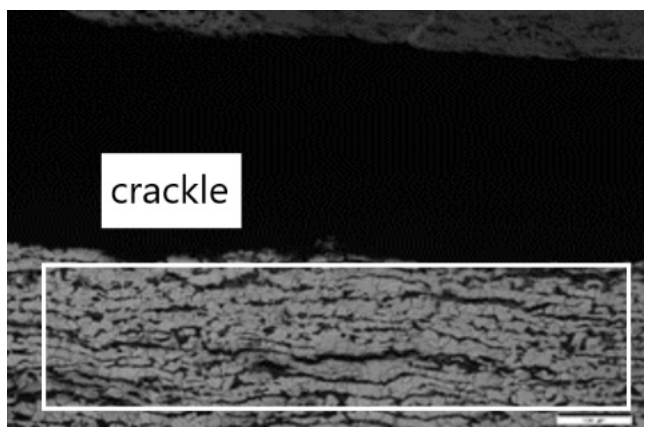

a. Corrosion pit

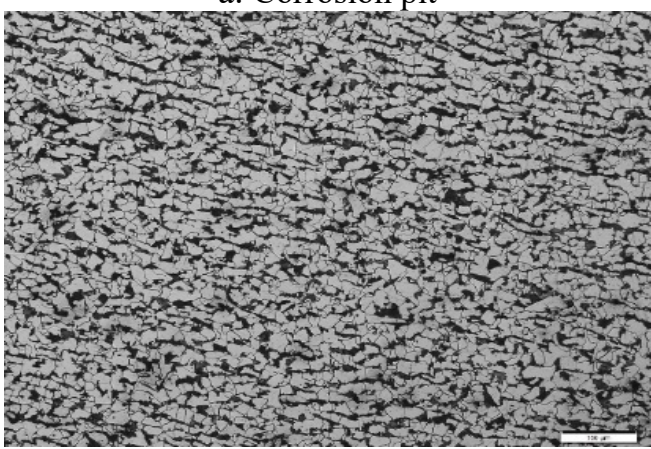

b. Center of wall thickness

Fig. 6. Metallographic structure of sample

\subsection{XRD phase analysis of scaling corrosion products}

The scale corrosion products were scraped on the inner wall of the water wall pipe, and then ground to powder to make the XRD test sample. The phase analysis was carried out by smartlab XRD. The scale corrosion products of the samples contain $\mathrm{Fe}_{2} \mathrm{O}_{3}, \mathrm{Fe}_{3} \mathrm{O}_{4}$, $\mathrm{NiCr} 0.5 \mathrm{Mn} 1.5 \mathrm{O} 4$. The results of phase analysis show that the scale is iron oxide scale (the magnetic $\mathrm{Fe}_{3} \mathrm{O}_{4}$ iron is the main one, and the mixture with $\mathrm{Fe}_{2} \mathrm{O}_{3}$ iron).

\section{Discussion and analysis}

The corrosion part of water wall pipe analyzed in this test occurred in the inner wall of the pipe to the fire side, and formed multiple corrosion pits under the scale of the inner wall of the pipe, which is typical corrosion under the scale. Under scale corrosion is a common local corrosion phenomenon of thermal power boiler. When the inner surface of the pipe on the heating surface of boiler is attached with scale, serious corrosion will occur under it, which is called under scale corrosion or under sediment corrosion. There are many reasons for corrosion under scale, such as poor water quality, improper adjustment of the flame center of boiler, too high heat load in some parts of the pipe, and poor boiler water circulation condition, etc.

The microstructure of the water wall tube was observed. It was found that the microstructure of the matrix was ferrite and pearlite, and no abnormality was found. However, there were decarburization phenomena in different degrees in the corrosion pit, i.e. the inner wall of the pipe, and a large number of microcracks were distributed along the grain boundary. The yield strength of the samples is lower than the standard requirements and is also related to the decarbonization of the microstructure found in metallographic examination. Judging from the above characteristics, the corrosion of water wall pipe belongs to acid corrosion (hydrogen corrosion) under scale. Acid corrosion under scale is due to the metal surface scaling, because of their poor thermal conductivity, the temperature of the pipe wall under the scale will rise, the furnace water infiltrating under the scale will be concentrated sharply, and it cannot be mixed with the furnace water in the pipe. As a result, the concentration of impurities in the furnace water under the scale becomes higher, and the cathode generates atomic hydrogen, which makes the metal pipe wall and scale concentrate a lot Hydrogen, which occurs under scale, is usually on the fire side.

The corrosion under acid scale (hydrogen corrosion) is characterized by the corrosion under dense scale, the decarbonization occurs locally in the pipe and micro cracks are formed along the grain boundary. Although the corrosion under acid scale occurs in the water wall pipe in this test, it is not yet to be judged from the decarbonization degree, crack morphology and pipe mechanical properties data. However, attention should be paid to this stage. If the pipe is not replaced in time, the corrosion will make the wall of the pipe at the fire side seriously thinner, the effective area of the pipe will be reduced, the strength, toughness and plasticity of the metal will be reduced sharply, and then a large area of water wall explosion will occur.

According to the data provided by the power plant, the boiler has the condition of long-term water quality not up to standard and acid pickling in time. The large area corrosion of water wall has direct relationship with the unqualified water quality of boiler, and ensuring the quality of boiler water supply is the key factor to avoid water wall corrosion.

\section{Conclusion and suggestion}

(1) The results of the experiment can be used to judge that the corrosion of the water wall is under scale corrosion.

(2) In order to avoid the corrosion aggravation and cause large area hydrogen corrosion, it is suggested to determine the area of the seriously corroded pipe row and replace it by nondestructive testing and pipe cutting test.

(3) The power generation enterprises shall regularly guarantee the quality of boiler water supply and acid pickling according to the standard requirements. 


\section{Reference}

1. Zhao Hai-rui. Study on the type and mechanism of corrosion under boiler scale $[\mathrm{J}]$. Science and technology wind, 2015 (04): 140

2. Zhang Wei-wei. Cause analysis and prevention measures of corrosion under the drum scale of a horizontal fast loading steam boiler [J]. Jiangxi building materials, 2017 (07): 268

3. Xu Song. Discussion on corrosion under scale at the bottom of dzl2 / 4-1.0-w II boiler drum [J]. Equipment manufacturing technology, 2013 (09): 79$80+83$

4. Liu Xi-feng. Failure analysis of industrial boilers in use - corrosion mechanism under scale and case analysis $[\mathrm{J}]$. Henan science and technology, 2010 (21): 66-67

5. ASME sa-210/ sa-210m medium carbon steel tubes for boilers and superheaters [S] ASME Committee on boiler and pressure vessel materials 2002

6. Li Jun-xi. Harm, preventive measures and cleaning methods of industrial boiler scale [J]. Modern enterprise education, 2006 (9): 145

7. DL / T438-2016 interpretation of metal technical supervision regulations for thermal power plants [S]. Beijing: China Electric Power Press, 2017 